\title{
The Dehydroepiandrosterone Awakening Response as a Possible Index of Subjective Sleep Quality
}

\author{
Masako Hasegawa-OhiRA, ${ }^{*}$ Kazumichi Suguri, ${ }^{* *}$ Shusaku Nomura ${ }^{* *}$
}

\begin{abstract}
The dynamics of dehydroepiandrosterone (DHEA) and cortisol secretion from sleep through awakening were examined by collecting and analyzing saliva samples using a novel method. Sixteen male university students who voluntarily participated in this study were instructed to sleep from $0000 \mathrm{~h}$ to $0600 \mathrm{~h}$, and their salivary cortisol and DHEA concentrations were evaluated during sleep until $1 \mathrm{~h}$ after awakening. We observed a marked elevation in DHEA upon awakening, referred to as the DHEA awakening response (DAR), which peaked at a different time from that of cortisol awakening response (CAR). Furthermore, DAR correlated positively with the subject's subjective quality of sleep, whereas cortisol concentration did not. Therefore, the DAR may be an index of subjective sleep quality.
\end{abstract}

Keywords: DHEA, cortisol, DHEA awakening response, cortisol awakening response, sleep quality.

Adv Biomed Eng. 5: pp. 132-136, 2016.

\section{Introduction}

The cortisol awakening response (CAR) is characterized by a rapid increase in cortisol concentration in the saliva for 30-40 min upon awakening [1]. This response is commonly used in psychoneuroendocrinology studies [2], since CAR reflects the activity of the hypothalamic-pituitary-adrenal (HPA) axis as well as changes in the degree of psychological stress. For example, CAR increases when individuals are in a highly stressed or fatigued state [3]. On the other hand, the response decreases in patients with post-traumatic stress disorder [4] and chronic fatigue syndrome [5]. Therefore, temporary or mid-term stress increases CAR, while prolonged stress decreases it.

Similar to cortisol, dehydroepiandrosterone (DHEA) is a hormone secreted from the adrenal cortex, and its levels change in relation to stress and the state of depression [6]. Particularly, salivary DHEA level and the cortisol/DHEA ratio in the morning are indicators of psychological and health status in the majority of the population. They are also associated with cognitive function, anxiety, and negative mood in older men [7] and predict the persistence of major depression in adolescents [8]. Therefore, it is important to quantify the levels of both hormones upon awakening.

Unlike CAR, no post-awakening peak of DHEA secretory activity has been observed [9]. However, the secretion of DHEA and other hormones may be affected immediately upon awakening, and exploring the DHEA secretory changes from sleep through awakening may provide useful information. Therefore,

This study was presented at the Symposium on Biomedical Engineering 2016, Asahikawa, September, 2016.

Received on July 22, 2016; revised on September 10, 2016; accepted on October 16, 2016.

* Faculty of Education, Shiga University, Otsu, Japan.

** Faculty of Engineering, Nagaoka University of Technology, Nagaoka, Japan.

\# 2-5-1 Hiratsu, Otsu, Shiga 520-0862, Japan.

E-mail: ohira@edu.shiga-u.ac.jp this study used a novel method to collect saliva samples intermittently during sleep, while also using conventional methods to collect saliva samples before sleep and after awakening to investigate the dynamics of DHEA and cortisol secretion throughout the sleeping and awakening periods.

\section{Methods}

\subsection{Subjects}

This study included 16 male university students [mean age (standard deviation), 22.6 (1.0) years; mean body mass index (standard deviation), $22.1(3.5) \mathrm{kg} / \mathrm{m}^{2}$ ] who provided written informed consent to participate. All subjects were healthy volunteers who were not taking any prescription medications.

This study was conducted in accordance with the ethical principles of the Helsinki Declaration and after obtaining informed consent from each subject. The study was approved by the ethics committee of Nagaoka University of Technology.

\subsection{Experimental procedures}

Before the study, the subjects underwent sleep control training at home. They were asked to go to bed at $0000 \mathrm{~h}$ and wake up after sleeping for at least $6 \mathrm{~h}$. This pattern was continued for at least 3 days before study initiation. Furthermore, considering the effects on secretion of salivary biochemical substances, the subjects were prohibited from drinking alcohol beginning the day before the study, and from eating, drinking, smoking, or performing rigorous exercise from $1 \mathrm{~h}$ before $(2100 \mathrm{~h})$ study initiation to after study completion $(0700 \mathrm{~h}$ the following day). During the study, the subjects were instructed to sleep at $0000 \mathrm{~h}$ and were woken up at $0600 \mathrm{~h}$ (Fig. 1) by an investigator. In order to control the awakening time, the subjects were not informed of the time in advance.

An investigator monitored the participant all through the experiment on-site using an infrared camera and also recorded video images. In case that nocturnal awakening was confirmed (did not happen frequently and desire to urinate was the primary reason), the experiment was terminated and the data set was abandoned. The subject was not included in this study. In other words, none of the 16 participants analyzed in this study had nocturnal awakening. 


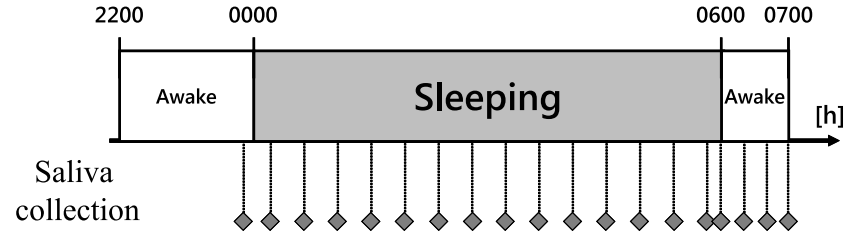

Fig. 1 Schematic presentation of the protocol.

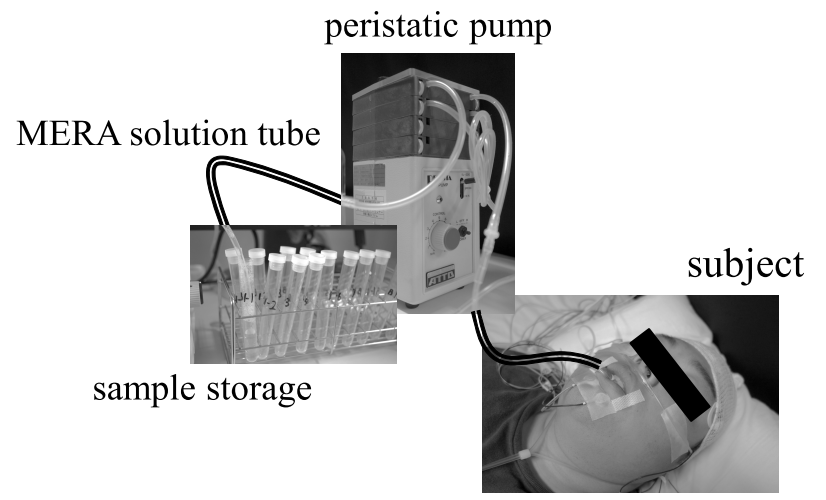

Fig. 2 Saliva collection system comprised of a suction mechanism and a collection device.

On the day of the study, the subjects were instructed to arrive at the laboratory by $2200 \mathrm{~h}$. Upon arrival, explanations of the study were given and informed consent forms were collected. The fitting and operation of the measurement devices were checked. Each subject slept in an air-conditioned laboratory (mean room temperature, $22^{\circ} \mathrm{C}$; mean humidity, $55 \%$ ).

\subsection{Measurement}

\subsubsection{Saliva collection}

For continuous collection of saliva during sleep, a saliva collection system comprised of a suction mechanism (a custom-made mouth piece) and a collection device (a peristaltic high-flow pump; SJ-1211H Peristapump ${ }^{\circledR}$, ATTO, Tokyo, Japan) was designed (Fig. 2). Using this system, small amounts of each subject's saliva could be collected noninvasively without disturbing sleep, thus facilitating the study of temporal changes in hormone concentrations during sleep. The mouthpiece (The Doctor's ${ }^{\circledR}$ NightGuard $^{\mathrm{TM}}$ Advanced Comfort ${ }^{\mathrm{TM}}$ Dental Protector, Prestige Brands Holdings, Inc., Tarrytown, NY, USA) was molded after the subject entered the laboratory, and a proper fit was confirmed. A Mera sustained saliva suction tube (SP-2, SENKO Medical Instrument Manufacturing Co. Ltd., Tokyo, Japan) was affixed to the molded mouthpiece and a peristaltic pump was connected via a silicone tube. To prevent blood accumulation in the oral cavity, the top of the Mera sustained saliva suction tube was secured with cotton wool. Although the use of cotton could interfere with the biochemical determination of some salivary hormones [10], this does not apply in this case as the cotton wool at the top of the Mera tube is filled with saliva that already exists in the mouth during the preparation period. After all, the design of our saliva collection method is not substantially different from the passive drool technique as both techniques collect "raw saliva."
Table 1 Results [mean (SD)] of Oguri-ShirakawaAzumi sleep inventory.

\begin{tabular}{lc}
\hline \hline & scores \\
\hline $\begin{array}{l}\text { Factor 1: } \\
\text { Sleepiness upon awakening }\end{array}$ & $15.6(5.2)$ \\
Factor 2: & \\
Falling asleep and sustainment of sleep & $15.4(5.9)$ \\
$\begin{array}{l}\text { Factor 3: } \\
\text { Frequency of dreaming }\end{array}$ & $29.8(3.7)$ \\
$\begin{array}{l}\text { Factor 4: } \\
\text { Recovery from fatigue }\end{array}$ & $17.7(6.7)$ \\
$\begin{array}{l}\text { Factor 5: } \\
\text { (Subjective) length of sleep }\end{array}$ & $17.9(5.7)$ \\
\hline \hline
\end{tabular}

Saliva was collected at 19 time points: $10 \mathrm{~min}$ before bedtime; every $30 \mathrm{~min}$ for the first $4 \mathrm{~h}$ during sleep; every $20 \mathrm{~min}$ for $2 \mathrm{~h}$ before awakening for more accurate observation of the awakening response; and immediately, $20 \mathrm{~min}$, $40 \mathrm{~min}$, and $60 \mathrm{~min}$ after awakening. During sleep, saliva was collected using the peristaltic pump described above. Before sleep and after awakening, naturally secreted saliva accumulated during a 3-min period was collected using a straw.

The collected saliva samples were maintained at $-25^{\circ} \mathrm{C}$, and cortisol and DHEA concentrations were quantitatively analyzed using enzyme-linked immunosorbent assays (ELISAs; cortisol: high-sensitivity salivary cortisol enzyme immunoassay kit, Salimetrics LLC., State College, PA, USA; DHEA: high-sensitivity salivary DHEA enzyme immunoassay kit, Salimetrics LLC.).

\subsubsection{Psychological parameters}

The subjects were asked to complete the Oguri-Shirakawa-Azumi sleep inventory (OSA) [11] immediately after awakening to record their subjective quality of sleep. This sleep questionnaire comprises 16 items, including factor 1: sleepiness upon awakening (higher score indicates less sleepiness, i.e. more alert); factor 2: falling asleep and sustainment of sleep; factor 3: frequency of dreaming (higher score indicates few or no dreams); factor 4: recovery from fatigue; and factor 5: (subjective) length of sleep.

\section{Results}

The OSA scores are shown in Table $\mathbf{1 .}$

With regard to salivary hormones, little changes in cortisol and DHEA concentrations were observed before bedtime and during sleep. Immediately after awakening, both concentrations increased to reach their respective peaks (Fig. 3). However, the time taken for each hormone to peak differed. The cortisol concentration increased rapidly approximately 15-30 min after awakening and reached a peak at $40 \mathrm{~min}$. On the other hand, the DHEA concentration increased rapidly upon awakening reaching a peak immediately after awakening [mean (standard deviation) of DHEA concentration averaged for $1 \mathrm{~h}$ before awakening (0500 to $0600 \mathrm{~h}$ ): 90.7 (97.3) pg/mL, versus the concentration just after awakening: 582.6 (375.2) $\mathrm{pg} / \mathrm{mL} ; p<0.001$, paired $t$-test] (Fig. 3). To examine the correlation between subjective quality of sleep and hormone concentration after awakening, the area under the curve with respect to ground (AUCG) [12] of DHEA and cor- 


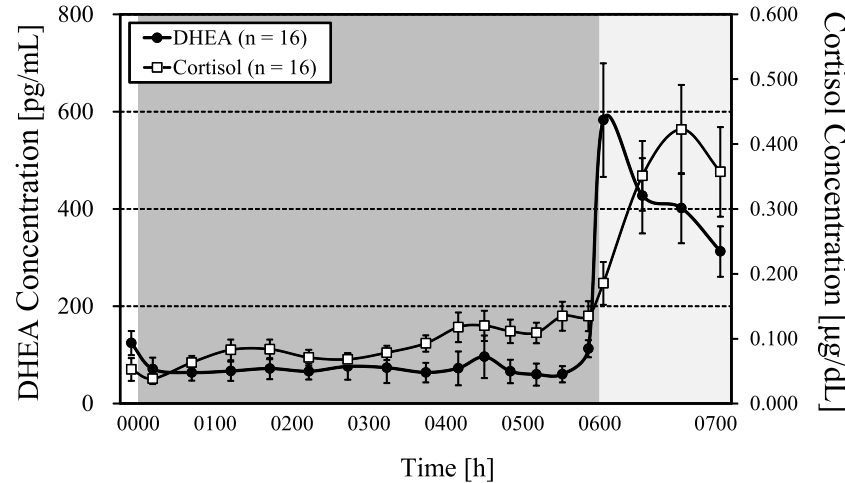

Fig. 3 Salivary dehydroepiandrosterone (DHEA) and cortisol concentrations during sleep in male students (mean \pm standard error). The dark grey shaded area indicates sleep, while the light grey shaded area indicates the hour after awakening.

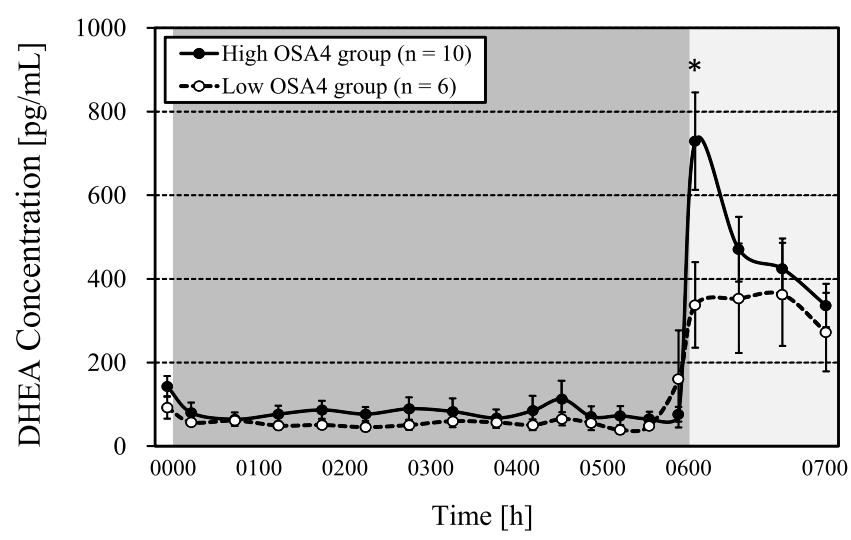

Fig. 4 Salivary dehydroepiandrosterone (DHEA) concentration during sleep in male students (mean \pm standard error) stratified by the score of factor 4 of the Oguri-Shirakawa-Azumi sleep inventory (OSA4; recovery from fatigue). The dark grey shaded area indicates sleep, while the light grey shaded area indicates the hour after awakening. (Student's $t$-test: ${ }^{*} p<0.05$ vs. Low OSA4 group).

tisol concentrations after awakening were calculated. A significant correlation was observed between the AUCG of DHEA and OSA factor 3 score (frequency of dreaming; $r=0.55, p<0.05$ ), and between the AUCG of DHEA and OSA factor 4 score (recovery from fatigue; $r=0.60, p<0.05$ ). However, the concentration of cortisol did not correlate significantly with the subjective quality of sleep (assessed using the OSA).

Next, the subjects were divided into two groups according to their responses to OSA factor 4: a high OSA4 group (OSA factor 4 score $>16.0, n=10$ ) and a low OSA4 group (OSA factor 4 score $\leq 16.0, n=6$ ). The mean (standard deviation) OSA factor 4 scores in the high and low OSA4 groups were 21.7 (2.8) and 11.1 (6.1), respectively, with a significant difference between the two groups ( $p<0.01$, Student's $t$-test). As illustrated in Fig. 4, a characteristic difference in DHEA secretion at awakening was observed. Specifically, the DHEA concentration just after awakening $(0600 \mathrm{~h})$ in the high OSA4 group [mean (standard deviation), $729.4(368.7) \mathrm{pg} / \mathrm{mL}$ ] was significantly higher than that in the

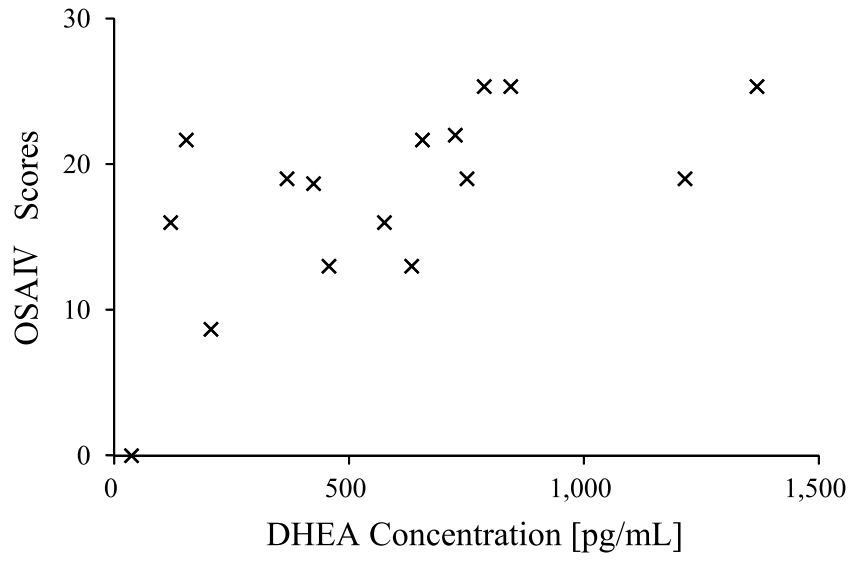

Fig. 5 Relation between salivary dehydroepiandrosterone (DHEA) concentration and score of factor 4 of the Oguri-ShirakawaAzumi sleep inventory (OSA4; recovery from fatigue).

low OSA4 group [337.9 (250.5) pg/mL; $p<0.05$, Student's $t$-test;] (Fig. 4). Furthermore, a significant correlation between DHEA concentration just after awakening and OSA factor 4 score was observed $(r=0.62, p<0.01)($ Fig. 5).

\section{Discussion}

Under the condition of no nocturnal awakening in all the subjects studied, the OSA scores presented in Table $\mathbf{1}$ are comparable to another experimental sleep study employing polysomnography (PSG) measurement [13]. Thus, in terms of subjective sleep quality, our saliva collection method would not have placed a burden on the subjects.

Although a previous study suggested no evidence of stimulated burst of DHEA secretory activity upon awakening [9], our novel saliva collection method that allows measurement of hormonal secretion serially through the sleeping and awakening periods demonstrated a significant enhancement in DHEA secretion upon awakening. It should be noted that this DHEA profile was not a result of the difference in methodology of saliva collection; i.e., our novel method during sleep and passive drool after awakening, as both methods essentially collect raw saliva, as mentioned above. Actually, the levels of biomarkers showed continuous profiles regardless of saliva collection method.

We termed this conspicuous DHEA elevation at awakening as the DHEA awakening response (DAR), as opposed to the CAR. AUC of the DAR correlated positively with a low dream frequency (OSA factor 3 ) and subjective recovery from fatigue (OSA factor 4). High score for factor 3 indicates few dreams and no nightmare, and hence good quality of sleep. High score for factor 4 incontrovertibly represents good quality of sleep. Furthermore, a stratified analysis using factor 4 score showed a significant and positive correlation with DHEA secretion at awakening. Therefore, the DAR may be related to sleep quality, especially recovery from fatigue.

The DHEA/cortisol ratio has been reported to associate with depression or other mental disease [14]. In this study also, there was a positive correlation between OSA factor 4 score and the ratio of post-awakening AUCG of DHEA to that of cortisol ( $r=$ $0.57, p<0.05)$. However, we assume that this correlation was attributed to DHEA, because there was no significant correlation 
between OSA and CAR.

Although the CAR is a promising index of mid- to long-term stress and an individual's health status [2,15], the subjects of this study were healthy males with no mental illnesses. This would explain our finding of no correlation between the CAR level and subjective quality of sleep. Our novel finding suggests that the DAR may be a good index of sleep quality, and may reflect shortterm stress and an individual's health status.

Our novel saliva collection method successfully demonstrated the impact of awakening on DHEA secretion. This method may also allow accurate measurement of CAR levels. For CAR measurements, compliance and timeliness are indispensable [2], and any deviation from saliva collection immediately after awakening impedes confirmation of the awakening response [16, 17]. Furthermore, the changes in salivary cortisol concentration observed during sleep (Fig. 1) in this study differed from the reported temporal changes in serum cortisol concentration during sleep [18]. The serum cortisol concentration increased intermittently during sleep but did not increase markedly upon awakening, contrary to the finding in saliva during the CAR. Conventional experiments in which blood is collected through the night (so-called isolation experiments) place the subjects under mental stress and may have a physiological impact caused by blood collection. The evaluation method used in this study placed very little burden on the subjects, and there was no nocturnal awakening. Therefore, the saliva collection system designed for this study proved useful for verifying the patterns of hormone secretion during sleep and after awakening.

For the DAR to be a useful tool for measuring hormone secretion in psychoneurotic studies, a population-based study that assesses subject's brain waves (in various sleep stages), gender, age, and health condition is required. Therefore, further clinical studies are required to clarify these issues.

\section{Conclusion}

The aim of this study was to determine the dynamics of dehydroepiandrosterone (DHEA) and cortisol secretion from sleep through awakening using a novel saliva collection method. Our results indicated that the DHEA concentration upon awakening was elevated, which we refer to as the DHEA awakening response (DAR). The peak DAR response was different from the peak cortisol awakening response. Additionally, the DAR, but not the cortisol awakening response, correlated positively with the subjects' subjective quality of sleep, suggesting that the DAR may be a potential index of subjective sleep quality.

\section{Acknowledgement}

This work was partially supported by the Japan Society for the Promotion of Science (JSPS) KAKENHI Grant Number 23680023 and $15 \mathrm{H} 02767$.

\section{References}

1. Pruessner JC, Wolf OT, Hellhammer DH, Buske-Kirschbaum A, von Auer K, Jobst S, Kaspers F, Kirschbaum C: Free cortisol concentrations after awakening: a reliable biological marker for the assessment of adrenocortical activity. Life Sci. 61(26), 25392549, 1997.

2. Clow A, Thorn L, Evans P, Hucklebridge F: The awakening cortisol response: methodological issues and significance. Stress.
7(1), 29-37, 2004.

3. Pruessner JC, Hellhammer DH, Kirschbaum C: Burnout, perceived stress, and cortisol responses to awakening. Psychosom Med. 61(2), 197-204, 1999.

4. Morris MC, Compas BE, Garber J: Relations among posttraumatic stress disorder, comorbid major depression, and HPA function: a systematic review and meta-analysis. Clin Psychol Rev. 32(4), 301-315, 2012.

5. Nijhof SL, Rutten JM, Uiterwaal CS, Bleijenberg G, Kimpen JL, Putte EM: The role of hypocortisolism in chronic fatigue syndrome. Psychoneuroendocrinology. 42, 199-206, 2014.

6. Michael A, Jenaway A, Paykel ES, Herbert J: Altered salivary dehydroepiandrosterone concentrations in major depression in adults. Biol Psychiatry. 48(10), 989-995, 2000.

7. van Niekerk JK, Huppert FA, Herbert J: Salivary cortisol and DHEA: association with measures of cognition and well-being in normal older men, and effects of three months of DHEA supplementation. Psychoneuroendocrinology. 26(6), 591-612, 2001.

8. Goodyer IM, Herbert J, Tamplin A: Psychoendocrine antecedents of persistent first-episode major depression in adolescents: a community-based longitudinal enquiry. Psychol Med. 33(4), 601-610, 2003.

9. Hucklebridge F, Hussain T, Evans P, Clow A: The diurnal patterns of the adrenal steroids cortisol and dehydroepiandrosterone (DHEA) in relation to awakening. Psychoneuroendocrinology. 30(1), 51-57, 2005.

10. Shirtcliff, EA, Granger DA, Schwartz E, Curran MJ: Use of salivary biomarkers in biobehavioral research: cotton-based sample collection methods can interfere with salivary immunoassay results. Psychoneuroendocrinology. 26(2), 165-173, 2001.

11. Yamamoto Y, Tanaka H, Takase M, Yamazaki K, Azumi K, Shirakawa S: Standardization of revised version of OSA sleep inventory for middle age and aged. Brain Science and Mental Disorders. 10, 401-409, 1999.

12. Pruessner JC, Kirschbaum C, Meinlschmid G, Hellhammer DH: Two formulas for computation of the area under the curve represent measures of total hormone concentration versus time-dependent change. Psychoneuroendocrinology. 28(7), 916-931, 2003.

13. Igasaki T, Taniyama Y, Matsuda $\mathrm{Y}$, Murayama N: Can quality of sleep be evaluated from hypnogram?-Estimation of factor score of Oguri-Shirakawa-Azumi sleep inventory by artificial neural network. Proc. the Information Systems International Conference. 373-378, 2013.

14. Young AH, Gallagher P, Porter RJ: Elevation of the cortisol-dehydroepiandrosterone ratio in drug-free depressed patients. Am J Psychiatry. 159(7), 1237-1239, 2002.

15. Adam EK, Hawkley LC, Kudielka BM, Cacioppo JT: Day-to-day dynamics of experience--cortisol associations in a population-based sample of older adults. Proc Natl Acad Sci U S A. 103(45), 17058-17063, 2006.

16. Dockray S, Bhattacharyya MR, Molloy GJ, Steptoe A: The cortisol awakening response in relation to objective and subjective measures of waking in the morning. Psychoneuroendocrinology. 33(1), 77-82, 2008.

17. Okun ML, Krafty RT, Buysse DJ, Monk TH, Reynolds CF, 3rd Begley A, Hall M: What constitutes too long of a delay? Determining the cortisol awakening response (CAR) using self-report and PSG-assessed wake time. Psychoneuroendocrinology. 35(3), 460-468, 2010.

18. Spiegel K, Leproult R, L'hermite-Balériaux M, Copinschi G, Penev PD, Van Cauter E: Leptin concentrations are dependent on sleep duration: relationships with sympathovagal balance, carbo- 
hydrate regulation, cortisol, and thyrotropin. J Clin Endocrinol Metab. 89(11), 5762-5771, 2004.

\section{Masako HaSEgawa-OHIRA}

Masako Hasegawa-OHIRA is an Assistant Professor at Shiga University, Japan. She received her Ph.D. in Medicine from the Graduate School of Medicine, Osaka University, in 2011. Her research interests include Psychoneuroendocrinology, and Medical and Biological Engineering.

\section{Kazumichi SugurI}

Kazumichi SugurI is a master course student in the faculty of Management and Information Systems Engineering, Nagaoka University of Technology, Japan. His research interests include Ambient Biomedical Engineering.

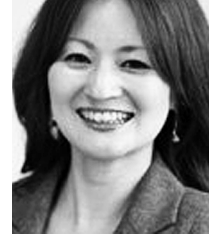

\section{Shusaku Nomura}

Shusaku Nomura is an Associate Professor at Nagaoka University of Technology, Japan. He received his Ph.D. in Science from the Graduate School of Science and Technology, Kobe University, in 2001. His research interests include Ambient Biomedical Engineering, Psychoneuroendocrinology, and Kansei Physiology.
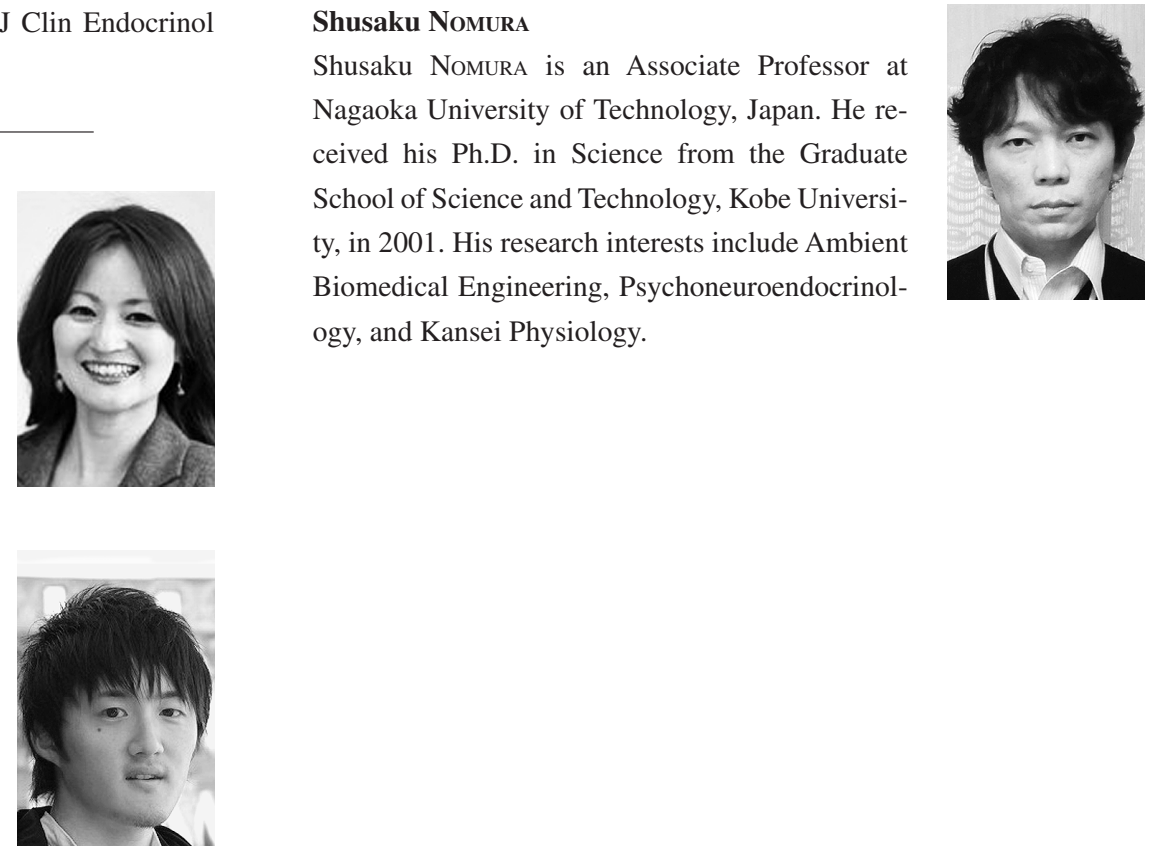